\title{
A free boundary problem for a coupled system of elliptic, hyperbolic, and Stokes equations modeling tumor growth
}

\author{
AVNER FRIEDMAN ${ }^{\dagger}$ \\ Department of Mathematics, The Ohio State University, Columbus, OH 43210, USA
}

[Received 27 July 2005 and in revised form 10 January 2006]

\begin{abstract}
We consider a tumor model with three populations of cells: proliferating, quiescent, and necrotic. Cells may change from one type to another at a rate which depends on the nutrient concentration. We assume that the tumor tissue is a fluid subject to the Stokes equation with sources determined by the proliferation rate of the proliferating cells. The boundary of the tumor is a free boundary held together by cell-to-cell adhesiveness of intensity $\gamma$. Thus, on the free boundary the stress tensor $T$ and the mean curvature $\kappa$ are related by $T \vec{n}=-\gamma \kappa \vec{n}$ where $\vec{n}$ is the outward normal. We prove that the coupled system of PDEs for the densities of the three types of cells, the nutrient concentration, and the fluid velocity and pressure have a unique smooth solution, with a smooth free boundary, for a small time interval.
\end{abstract}

2000 Mathematics Subject Classification: 35L85, 35Q30, 35Q80, 35R35, 92 C99.

Keywords: Tumor growth; free boundary problems; hyperbolic equations; Stokes equation.

\section{The model}

In this paper we consider a model of tumor growth described in terms of elliptic and hyperbolic PDEs coupled to the Stokes equation. The model involves three populations of cells: proliferating cells with density $r(x, t)$, quiescent cells with density $q(x, t)$, and necrotic cells with density $n(x, t)$. Proliferating cells change into quiescent cells at a rate $K_{Q}(c)$, which depends on the concentration $c(x, t)$ of the nutrients within the tumor. Similarly, quiescent cells become proliferating cells at a rate $K_{R}(c)$, and they become necrotic at a rate $K_{N}(c)$. Proliferating cells have a proliferation (or growth) rate $K_{B}(c)$. Naturally,

$$
\begin{aligned}
& K_{R}(c), K_{Q}(c) \text {, and } K_{N}(c) \text { are positive-valued functions; } \\
& K_{Q}(c) \text { and } K_{N}(c) \text { are decreasing in } c, \text { while } \\
& K_{R}(c) \text { and } K_{B}(c) \text { are increasing in } c .
\end{aligned}
$$

The function $K_{B}(c)$ represents the balance between birth and death of the proliferating cells. We assume that

$$
K_{B}(\tilde{c})=0 \quad \text { for some } \tilde{c}>0
$$

$\tilde{c}$ is a critical nutrient concentration: if $c>\tilde{c}$ then the population of proliferating cells grows, whereas if $c<\tilde{c}$ then the population of proliferating cells decreases.

We also assume that the necrotic cells degrade, and are removed from the tumor, at a constant rate $K_{O}$.

†E-mail: afriedman@mbi.osu.edu 
We next need to introduce a constitutive law for the tissue. Most tumor models assume that the tissue has the structure of a porous medium for which Darcy's law applies (see, for example, [5], [21]). There are however tumors for which the tissue is more naturally modeled as a fluid. For example, in early stages of breast cancer the tumor is confined to the duct of a mammary gland, which consists of epithelial cells, a meshwork of proteins, and extracellular fluid. Several recent papers on ductal carcinoma in the breast use the Stokes equation in their mathematical model [10][13]. If we denote the fluid velocity by $\vec{v}=\left(v_{1}, v_{2}, v_{3}\right)$ and the fluid pressure by $p$, then the constitutive law is

$$
\sigma_{i j}=-p \delta_{i j}+2 v\left(e_{i j}-\frac{1}{3} \bar{\Delta} \delta_{i j}\right)
$$

where $\sigma_{i j}$ is the stress tensor, $p=-\frac{1}{3} \sigma_{k k}$,

$$
e_{i j}=\frac{1}{2}\left(\frac{\partial v_{i}}{\partial x_{j}}+\frac{\partial v_{j}}{\partial x_{i}}\right)
$$

is the strain tensor, $\bar{\Delta}=e_{k k}=\operatorname{div} \vec{v}$ is the dilatation, and $v$ is the viscosity coefficient. If there are no body forces then

$$
\sum_{j=1}^{3} \frac{\partial \sigma_{i j}}{\partial x_{j}}=0 .
$$

We can rewrite this equation as the Stokes equation

$$
\nabla p-v \Delta \vec{v}-\frac{1}{3} v \nabla \operatorname{div} \vec{v}=0 \quad \text { in } \Omega_{t}, t>0
$$

where $\Omega_{t}$ is the tumor region.

We assume that the cells move with the fluid velocity $\vec{v}$. Then, by conservation of mass,

$$
\begin{aligned}
& \frac{\partial r}{\partial t}+\operatorname{div}(r \vec{v})=\left[K_{B}(c)-K_{Q}(c)\right] r+K_{R}(c) q, \\
& \frac{\partial q}{\partial t}+\operatorname{div}(q \vec{v})=K_{Q}(c) r-\left[K_{R}(c)+K_{N}(c)\right] q, \\
& \frac{\partial n}{\partial t}+\operatorname{div}(n \vec{v})=K_{N}(c) q-K_{O} n .
\end{aligned}
$$

We next assume that all the cells are of the same volume and mass, and that the total density of the cells is uniform throughout the tumor. Then, after normalization, we have

$$
r+q+n=1 \text {. }
$$

Summing up the three preceding conservation laws, we deduce that $\operatorname{div} \vec{v}=K_{B}(c) r-K_{O}$. This equation can be used to replace the conservation law for $n$. We now substitute $n=1-r-q$ into the expression for $\operatorname{div} \vec{v}$ and, together with the conservation laws for $r$ and $q$, we obtain the system

$$
\begin{aligned}
& \operatorname{div} \vec{v}=h(c, r, q) \quad \text { in } \Omega_{t}, t>0, \\
& \frac{\partial r}{\partial t}+\vec{v} \cdot \nabla r=f(c, r, q) \quad \text { in } \Omega_{t}, t>0 \text {, } \\
& \frac{\partial q}{\partial t}+\vec{v} \cdot \nabla q=g(c, r, q) \quad \text { in } \Omega_{t}, t>0,
\end{aligned}
$$


where

$$
\begin{aligned}
& h(c, r, q)=-K_{O}+\left[K_{B}(c)+K_{O}\right] r+K_{O} q, \\
& f(c, r, q)=\left[K_{B}(c)-K_{Q}(c)\right] r+K_{R}(c) q-r h(c, r, q), \\
& g(c, r, q)=K_{Q}(c) r-\left[K_{R}(c)+K_{N}(c)\right] q-q h(c, r, q) .
\end{aligned}
$$

The nutrient concentration $c$ is depleted as it is consumed by the live cells. We assume that it satisfies a quasi-stationary diffusion equation

$$
\Delta c-\lambda(r+q) c=0 \quad \text { in } \Omega_{t}, t>0,
$$

where $\lambda$ is a positive constant; in Section 4 we shall consider briefly also a parabolic equation for $c$.

We now turn to the boundary conditions at the boundary $\Gamma_{t}$ of $\Omega_{t}$. We assume that the tumor is held together by the forces of cell-to-cell adhesion with constant intensity $\gamma$; the role of $\gamma$ is discussed in [2]-[4]. Introducing the stress tensor $T=v\left(\nabla \vec{v}+(\nabla \vec{v})^{*}\right)-\left(p+\frac{2 v}{3} \operatorname{div} \vec{v}\right) I$ with components

$$
T_{i j}=v\left(\frac{\partial v_{i}}{\partial x_{j}}+\frac{\partial v_{j}}{\partial x_{i}}\right)-\delta_{i j}\left(p+\frac{2 v}{3} \operatorname{div} \vec{v}\right),
$$

we then have

$$
T \vec{n}=-\gamma \kappa \vec{n} \quad \text { on } \Gamma_{t}, t>0
$$

where $\vec{n}$ is the outward unit normal and $\kappa$ is the mean curvature $\left(\kappa>0\right.$ if $\Gamma_{t}$ is the surface of a convex body). We also assume the kinematic condition

$$
\vec{v} \cdot \vec{n}=V_{n} \quad \text { on } \Gamma_{t}, t>0,
$$

where $V_{n}$ is the velocity of the free boundary $\Gamma_{t}$ in the direction $\vec{n}$. Finally, we assume that

$$
c=\text { const }=\bar{c} \quad \text { on } \Gamma_{t}, t>0,
$$

where $\bar{c}$ is a time and space-independent constant. We note that no boundary conditions are needed for $r$ and $q$, since (by (1.10p) the characteristic curves initiating at $\Gamma_{0}$ will remain on $\Gamma_{t}$ for all $t$.

The system (1.3), (1.4), and (1.9) has six-dimensional kernel $V_{0}$ consisting of rigid motions

$$
\vec{v}_{0}=\vec{a}+\vec{b} \times \vec{x}, \quad p_{0}=0 .
$$

We must therefore add six scalar constraints; see [22]. These constraints can be written in the form

$$
\begin{aligned}
\int_{\Omega_{t}} \vec{v} \mathrm{~d} x & =\vec{A}(t), \\
\int_{\Omega_{t}} \vec{v} \times \vec{x} \mathrm{~d} x & =\vec{B}(t)
\end{aligned}
$$

where $\vec{A}(t), \vec{B}(t)$ are prescribed functions. 
Finally, we prescribe initial conditions:

$$
\left.\Omega\right|_{t=0}=\Omega_{0},\left.\quad r\right|_{t=0}=r_{0}(x),\left.\quad q\right|_{t=0}=q_{0}(x) .
$$

Note that, given $\Omega_{0}, r_{0}, q_{0}$, the function $c_{0}=\left.c\right|_{t=0}$ is determined by (1.8), (1.11), so that also $h_{0}=\left.h(c, r, q)\right|_{t=0}$ is determined. We can then solve (1.3), (1.9) (with div $\vec{v}=h_{0}$ ) with the constraints (1.12), (1.13), at $t=0$.

We assume that

$$
r_{0}(x) \geqslant 0, \quad q_{0}(x) \geqslant 0, \quad r_{0}(x)+q_{0}(x) \leqslant 1 \quad \text { in } \Omega_{0} .
$$

Since proliferating cells reside in nutrient rich regions and, in particular, near the boundary of the tumor, we shall assume that

$$
r_{0}(x)=1 \quad \text { on } \Gamma_{0} .
$$

We shall also assume that

$$
K_{Q}(\bar{c})=0,
$$

that is, proliferating cells do not become quiescent if the nutrient concentration is at its maximum $\bar{c}$.

REMARK 1.1 As in [7] one can easily show that for any solution of (1.3)-11.16],

$$
r \geqslant 0, \quad q \geqslant 0, \quad r+q \leqslant 1
$$

in fact $r+q+n \equiv 1$ and $n$, the density of necrotic cells, is $\geqslant 0$. Also $0 \leqslant c \leqslant \bar{c}$ by the maximum principle. Hence without loss of generality we may truncate the functions $h(c, r, q), f(c, r, q), g(c, r, q)$ for $r<0, q<0, c<0$ and $r, q$, and $c$ positive and large, so that these functions remain as smooth in their variables as the original functions and, at the same time, they have compact support.

REMARK 1.2 In view of (1.10) the characteristic curves of (1.5), (1.6) which start on $\Gamma_{0}$ will lie on $\Gamma_{t}$ for all $t$. Since $c=\bar{c}$ on $\Gamma_{t}$ and $K_{Q}(\bar{c})=0$, the unique solution of (1.5), 1.6) along $\Gamma_{t}$ with initial conditions 1.16 ) and $q_{0}=0$ (by (1.15) ) is $q \equiv 0, r \equiv 1$, and from 1.4, (1.7) we then get

$$
\left.\operatorname{div} \vec{v}\right|_{\Gamma_{t}}=\text { const }=K_{B}(\bar{c})
$$

The system 1.3 - 1.17 may be viewed as a problem for $(\vec{v}, p)$ coupled to a problem for $(c, r, q)$. In Section 2 we consider the subproblem for $(\vec{v}, p)$. Using results of Solonnikov [22] we establish existence, uniqueness, and regularity estimates. Next, in Section 3, we consider the elliptic-hyperbolic system for $(c, r, q)$ and, by means of the results established in Section 2, we define a mapping $(c, r, q) \mapsto S(c, r, q)$. We prove that if $0 \leqslant t \leqslant T$ where $T$ is sufficiently small then $S$ is a contraction, and its fixed point is the solution of the system (1.3)-(1.17). The free boundary $\Gamma_{t}$ and its first $t$-derivative are shown to be smooth functions in the spatial variables. Our proof of existence, uniqueness, and regularity for the solution of (1.3)-(1.17) does not use the special assumptions (1.1), 1.2), and (1.7); the proof is valid for general functions $h(c, r, q), f(c, r, q)$ and $g(c, r, q)$, provided div $\vec{v}=$ const on $\Gamma_{t}$ (as in 1.18$)$. In Section 4 we prove a few additional results and state some open problems. 


\section{Auxiliary results}

In this section we study the auxiliary problem

$$
\begin{array}{rlrl}
-v \Delta \vec{v}+\nabla p=\vec{f} & \left(\vec{f}=-\frac{v}{3} \nabla g\right) & \text { in } \Omega_{t}, t>0, \\
\operatorname{div} \vec{v}=g & \text { in } \Omega_{t}, t>0, \\
T(\vec{v}, p) \vec{n}=-\gamma \kappa \vec{n} & \text { on } \Gamma_{t}, t>0, \\
\vec{v} \cdot \vec{n} & =V_{n} \quad \text { on } \Gamma_{t}, t>0, \\
& \Omega_{0} \text { is given. }
\end{array}
$$

We introduce a basis $\vec{w}_{1}(x), \ldots, \vec{w}_{6}(x)$ in the six-dimensional space $V_{0}$ generated by $\vec{a}+\vec{b} \times \vec{x}$ where $\vec{a}, \vec{b}$ are any vectors in $\mathbb{R}^{3}$ :

$$
\begin{gathered}
\vec{w}_{1}=(1,0,0), \quad \vec{w}_{2}=(0,1,0), \quad \vec{w}_{3}=(0,0,1), \quad \vec{w}_{4}=\left(0,-x_{3}, x_{2}\right), \\
\vec{w}_{5}=\left(x_{3}, 0,-x_{1}\right), \quad \vec{w}_{6}=\left(-x_{2}, x_{1}, 0\right) .
\end{gathered}
$$

We can then write the constraints 1.12 ,, 1.13 in the form

$$
\left(\vec{v}, \vec{w}_{k}\right)=M_{k}(t) \quad(k=1, \ldots, 6)
$$

where $\left(\vec{v}, \vec{w}_{k}\right)=\int_{\Omega_{t}} \vec{v}(x, t) \cdot \vec{w}_{k}(x) \mathrm{d} x$, and the functions $M_{k}(t)$ are linearly dependent on the components $A_{j}(t), B_{j}(t)$ of $\vec{A}(t), \vec{B}(t)$. Note that

$$
\operatorname{div} \vec{w}_{k}=0 \quad \text { for all } k .
$$

We recall [19, Lemma 6.1] that

$$
\int_{\Gamma_{t}} \kappa \vec{w}_{k} \cdot \vec{n}=0 \quad(1 \leqslant k \leqslant 6) .
$$

We also have

$$
\int_{\Gamma_{t}} \vec{w}_{k} \cdot \vec{n}=0 \quad(1 \leqslant k \leqslant 6) .
$$

Indeed,

$$
\int_{\Gamma_{t}} \vec{w}_{k} \cdot \vec{n}=\int_{\Omega_{t}} \operatorname{div} \vec{w}_{k}=0 \text { by } 2.7 .
$$

Our treatment of the problem 2.17-2.6 will be similar to that of Solonnikov [22] who proved existence and uniqueness in the case div $\vec{v}=0$, that is, when $\vec{f}=0, g=0$. (An alternative proof was given by Günther and Prokert [20].) Accordingly, we shall replace the Eulerian variable $x$ by the Lagrangian variable $\xi$, where

$$
\vec{x}=\vec{\xi}+\int_{0}^{t} \vec{u}(\xi, \tau) \mathrm{d} \tau \equiv X(\xi, t), \quad \xi \in \Omega,
$$

$\Omega=\Omega_{0}$, and

$$
\vec{u}(\xi, t)=\vec{v}(X(\xi, t), t), \quad \tilde{p}(\xi, t)=p(X(\xi, t), t) ;
$$

for simplicity, we shall often denote $\vec{x}$ and $\vec{\xi}$ by $x$ and $\xi$. 
We introduce the notation

$$
|w|_{C^{m+\alpha}(\Omega)}=\sum_{|j| \leqslant m} \sup _{\Omega}\left|D^{j} w(x)\right|+\sum_{|j|=m} H_{\alpha, \Omega}\left(D^{j} w\right)
$$

where

$$
H_{\alpha, \Omega}(k)=\sup _{x, y \in \Omega} \frac{|k(x)-k(y)|}{|x-y|^{\alpha}}
$$

here $m$ is any integer $\geqslant 0$ and $\alpha \in(0,1)$. We set $\Gamma=\Gamma_{0}=\partial \Omega_{0}$, and similarly define $|w|_{C^{m+\alpha}(\Gamma)}$.

A function $w(x, t)$ is said to belong to $C\left(0, T ; C^{m+\alpha}(\Omega)\right)$ if $t \mapsto w(\cdot, t)$ is a continuous function from $[0, T]$ into $C^{m+\alpha}(\Omega)$, and we then define the norm

$$
|w|_{C\left(0, T ; C^{m+\alpha}(\Omega)\right)}=\sup _{0 \leqslant t \leqslant T}|w(\cdot, t)|_{C^{m+\alpha}(\Omega)} .
$$

Similarly we define the concept $w \in C^{1}\left(0, T ; C^{m+\alpha}(\Omega)\right)$.

We cover $\Gamma$ with a finite number of coordinate patches $\left(\eta_{1}, \eta_{2}, \eta_{3}\right)$ such that in each patch we can write $\eta_{1}=\varphi_{i}\left(\eta^{\prime}\right)$, where $\eta^{\prime}=\left(\eta_{2}, \eta_{3}\right)$ varies in some open set $\omega_{i}$. Suppose we can represent $\Gamma_{t}$ locally in the form

$$
\eta_{1}=\varphi_{i}\left(\eta^{\prime}, t\right), \quad \text { where } \quad \varphi_{i} \in C\left(0, T ; C^{m+\alpha}\left(\omega_{i}\right)\right) .
$$

Then we say that $\Gamma_{t}$ belongs to $C\left(0, T ; C^{m+\alpha}\right)$ and set

$$
\left|\Gamma_{t}\right|_{C\left(0, T ; C^{m+\alpha}\right)}=\sum_{i}\left|\varphi_{i}\right|_{C\left(0, T ; C^{m+\alpha}\left(\omega_{i}\right)\right)} .
$$

Similarly we define the concept $\Gamma_{t} \in C^{1}\left(0, T ; C^{m+\alpha}\right)$.

The system 2.1 -2.3 is an elliptic system in the Agmon-Douglas-Nirenberg sense, but the homogeneous system (with $\vec{f}=0, g=0$ ) has the 6-dimensional null space $V_{0}$. In order to secure uniqueness, we need to factor out the null space. This can be done, as in [22], by using the Schmidt lemma [24, Section 21]. Accordingly, we replace (2.1) by

$$
-v \Delta \vec{v}+\ell(\vec{v})+\nabla p=\vec{f} \quad\left(\vec{f}=-\frac{v}{3} \nabla g\right) \quad \text { in } \Omega_{t}, t>0,
$$

where

$$
\ell(\vec{v})=\sum_{k=1}^{6}\left[\left(\vec{v}, \vec{w}_{k}\right)-M_{k}(t)\right] \vec{w}_{k}(x)
$$

Then the system 2.11, 2.2, 2.3 has a unique solution. We can now state:

TheOREM 2.1 Let $\Gamma_{0} \in C^{m+3+\alpha}(m \geqslant 0,0<\alpha<1)$ and assume that, for some $T_{0}>0$, $g$ belongs to $C\left(0, T_{0} ; C^{m+1+\alpha}\left(\Omega_{0}\right)\right)$ and the $M_{k}(t)$ are continuous functions for $0 \leqslant t \leqslant T_{0}$. If $T$ is sufficiently small then there exists a unique solution $(\vec{v}, p)=(\vec{u}, p)$ to $(2.11),(2.2)-(2.5)$ such that $\Gamma_{t}$ belongs to $C\left(0, T ; C^{m+3+\alpha}\right) \cap C^{1}\left(0, T ; C^{m+2+\alpha}\right), \vec{u}(\xi, t)$ belongs to $C\left(0, T ; C^{m+2+\alpha}(\Omega)\right)$, and $\tilde{p}(\xi, t)$ belongs to $C\left(0, T ; C^{m+1+\alpha}(\Omega)\right)$; furthermore,

$$
\begin{aligned}
& |\vec{u}|_{C\left(0, T ; C^{m+2+\alpha}(\Omega)\right)}+|\tilde{p}|_{C\left(0, T ; C^{m+1+\alpha}(\Omega)\right)}+\left|\Gamma_{t}\right|_{C\left(0, T ; C^{m+3+\alpha}\right)} \\
& +\left|\Gamma_{t}\right|_{C^{1}\left(0, T ; C^{m+2+\alpha}\right)} \leqslant C\left\{|g|_{C\left(0, T ; C^{m+1+\alpha}(\Omega)\right)}+\sum_{k=1}^{6} \sup _{0 \leqslant t \leqslant T}\left|M_{k}(t)\right|\right\}
\end{aligned}
$$

where $C$ is a constant independent of $g$. 
Proof. We rewrite the system (2.11), 2.2)-(2.4) in the Lagrangian coordinates. As in [22], $\nabla_{x}$ can be written in the form $\sum_{j} A_{i j} \frac{d}{\partial \xi_{j}}$ where, if $\operatorname{div} \vec{v}=0$,

$$
A_{i j} \text { are cofactors of } \delta_{i j}+\int_{0}^{t} \frac{\partial u_{i}(\xi, \tau)}{\partial \xi_{j}} \mathrm{~d} \tau,
$$

and $T(\vec{v}, p)$ takes the form

$$
T_{u}(\vec{u}, \tilde{p})=-\left(\tilde{p}+\frac{2 v}{3} g\right) I+v S_{u}(\vec{u})
$$

where $S_{u}(\vec{u})$ is a first order differential operator with coefficients $A_{i j}$. In the present case, where $\operatorname{div} \vec{v}=g$, the determinant of the Jacobian $(\partial X / \partial \xi)$ is not identically equal to 1 in general,

$$
A_{i j} \text { are the elements of the Jacobian of the inverse transformation }
$$

(which depends on $g$ ), and some coefficients of the Stokes equation there will depend on $g(X(\xi, t), t)$ where $\xi$ is the Lagrangian coordinate. We also need to replace $\ell(\vec{v})$ by

$$
\tilde{\ell}(\vec{u})=\sum_{k=1}^{6}\left\{\int_{\Omega}\left[\vec{u}(\eta, t) \cdot \vec{w}_{k}(x(\eta, t))-M_{k}(t)\right] \operatorname{det}\left(\frac{\partial x}{\partial \eta}\right) \mathrm{d} \eta\right\} \vec{w}_{k}(x(\xi, t)) .
$$

We can nevertheless proceed as in [22] with minor changes. The proof is based on linearization and localization of the system written in the Lagrange variables (in the fixed domain $\Omega \times(0, T)$ ). A critical step is the careful study of a model problem in a half-space for an inhomogeneous system. In this step, the introduction of $\vec{f}$ and $g$ in the present case of 2.11, 2.2) does not cause any changes in the proof, so that Theorem 2 in [22], which deals with the model problem, extends to the present case.

Next we write the system, as in [22], in the form of a perturbation problem

$$
L(\vec{u}, \tilde{p})=\vec{F}(\vec{u}, \tilde{p}, t)
$$

where $L$ is a linear operator. As in [22], we can use the estimates for the model problem to derive existence, uniqueness and estimates for the linear problem $L(\vec{u}, \tilde{p})=\vec{F}$ for any given function $\vec{F}$. We then can solve 2.15 ) for $0 \leqslant t \leqslant T, T$ small, by a fixed point argument (using a contraction mapping, or successive approximations), and here again the fact that $\vec{f}$ and $g$ are non-zero functions makes for only trivial changes in the proof of [22] for the case $\vec{f}=0, g=0$.

Returning to the Eulerian coordinates, we then obtain the asserted solution of 2.11, 2.2 -2.5 with the estimate (2.13).

Observe that $\partial X(\xi, t) / \partial t=\vec{u}(\xi, t)$ belongs to $C\left(0, T ; C^{m+2+\alpha}(\Omega)\right)$. Hence $\partial A_{i j} / \partial t$ belongs to the same class. If we assume that

$$
g \in C^{1}\left(0, T ; C^{m+\alpha}(\Omega)\right), \quad M_{k} \in C^{1}(0, T),
$$

then we can formally differentiate the system for $\vec{u}(\xi, t), \tilde{p}(\xi, t)$ in $t$ and obtain an elliptic system for $\vec{u}_{t}, \tilde{p}_{t}$. Since $\Gamma_{t} \in C^{1}\left(0, T ; C^{m+2+\alpha}\right)$, the boundary conditions have sufficient regularity to ensure that

$$
\vec{u}_{t} \in C\left(0, T ; C^{m+1+\alpha}(\Omega)\right), \quad \tilde{p}_{t} \in C\left(0, T ; C^{m+\alpha}(\Omega)\right) .
$$


A rigorous proof of 2.17 can be obtained by working with finite differences in $t$, or, alternatively, by first establishing existence, uniqueness, and regularity of a solution $\left(\vec{u}^{*}, \tilde{p}^{*}\right)$ of the formally differentiated system (with $\Gamma_{t}$ being the free boundary established in Theorem 2.1 ) and then verifying that

$$
\begin{aligned}
& \vec{u}(\xi, t)=\vec{u}(\xi, 0)+\int_{0}^{t} \vec{u}^{*}(\xi, \tau) \mathrm{d} \tau, \\
& \tilde{p}(\xi, t)=\tilde{p}(\xi, 0)+\int_{0}^{t} \tilde{p}^{*}(\xi, \tau) \mathrm{d} \tau .
\end{aligned}
$$

We conclude:

THEOREM 2.2 If 2.16 holds then the solution established in Theorem 2.1 satisfies 2.17), and

$$
|\vec{u}|_{C^{1}\left(0, T ; C^{m+1+\alpha}\right)}+|\tilde{p}|_{C^{1}\left(0, T ; C^{m+\alpha}(\Omega)\right)} \leqslant C\left(\|g\|_{m+1+\alpha}+\|M\|\right)
$$

where the norm \|\|$_{m+1+\alpha}$ is defined by

$$
\|\varphi\|_{m+1+\alpha}=|\varphi|_{C\left(0, T ; C^{m+1+\alpha}(\Omega)\right)}+|\varphi|_{C^{1}\left(0, T ; C^{m+\alpha}(\Omega)\right)}
$$

and

$$
\|M\|=\sum_{k=1}^{6} \sup _{0 \leqslant t \leqslant T}\left[\left|M_{k}(t)\right|+\left|M_{k}^{\prime}(t)\right|\right] .
$$

We would like to replace 2.11 by 2.1 , that is, to show that $\ell(\vec{v})=0$. This is not possible for general $g(x, t)$. However, if $g(x, t)=h(c, r, q)$ where $h(c, r, q)$ is as in (1.7), then, as noted in Remark 1.2

$$
g(x, t)=\text { const } \quad \text { on } \Gamma_{t} .
$$

In preparation for this case we shall prove:

THEOREM 2.3 If 2.20 holds then in Theorem 2.1 we can replace 2.11 by 2.1 ; that is, $\ell(\vec{v})=0$ and the constraints 2.6) are satisfied.

Proof. We recall [20] the identity, for any $\vec{v}, \vec{w}, p$,

$$
\begin{aligned}
\frac{1}{2} \int_{\Omega_{t}} \sum\left(\frac{\partial v_{i}}{\partial x_{j}}+\frac{\partial v_{j}}{\partial x_{i}}\right)\left(\frac{\partial w_{i}}{\partial x_{j}}+\frac{\partial w_{j}}{\partial x_{i}}\right) \mathrm{d} x-\int_{\Omega_{t}} p \operatorname{div} \vec{w} \mathrm{~d} x \\
=\int_{\Omega_{t}}(-\Delta \vec{v}+\nabla p) \cdot \vec{w} \mathrm{~d} x-\int_{\Omega_{t}} \nabla(\operatorname{div} \vec{v}) \cdot \vec{w} \mathrm{~d} x+\int_{\Gamma_{t}} T(\vec{v}, p) \cdot \vec{w} \mathrm{~d} S
\end{aligned}
$$

Taking for $(\vec{v}, p)$ the solution established in Theorem 2.1 and $\vec{w}=\vec{w}_{\ell}$ as in 2.12 , and noting that the left hand side vanishes if $w=\vec{w}_{\ell}$ and the integral on $\Gamma_{t}$ vanishes by 2.3, 2.8, we obtain

$$
\int_{\Omega_{t}}(\vec{f}-\ell(\vec{v})) \cdot \vec{w}_{\ell} \mathrm{d} x-\int_{\Omega_{t}} \nabla g \cdot \vec{w}_{\ell} \mathrm{d} x=0 \quad\left(\vec{f}=-\frac{v}{3} \nabla g\right) .
$$

Since

$$
\int_{\Omega_{t}} \nabla g \cdot \vec{w}_{\ell}=\int_{\Gamma_{t}} g \vec{w}_{\ell} \cdot \vec{n}=\mathrm{const} \cdot \int_{\Gamma_{t}} \vec{w}_{\ell} \cdot \vec{n}=0
$$


by 2.20 and 2.9 , we get $\int_{\Omega_{t}} \ell(\vec{v}) \cdot \vec{w}_{\ell}=0$, that is,

$$
\sum_{k=1}^{6}\left[\left(\vec{v}, \vec{w}_{k}\right)-M_{k}(t)\right]\left(\vec{w}_{k}, \vec{w}_{\ell}\right)=0 \quad \text { for } \ell=1, \ldots, 6 .
$$

It follows that the expressions in brackets vanish. Thus the constraints 2.6 are satisfied, and 2.11) reduces to 2.1.

\section{The main result}

We shall need the following lemma.

LEMMA 3.1 Consider the hyperbolic system

$$
\begin{aligned}
\vec{w}_{t}+\left(\vec{b} \cdot \nabla_{x}\right) \vec{w} & =\vec{G}(x, t, \vec{w}) \quad \text { in } \mathbb{R}^{n} \times(0, T), \\
\left.\vec{w}\right|_{t=0} & =\vec{w}_{0} \quad \text { in } \mathbb{R}^{n},
\end{aligned}
$$

where $n \geqslant 1, \vec{w}=\left(w_{1}, w_{2}\right), \vec{G}=\left(G_{1}, G_{2}\right)$, and assume that

$$
\begin{gathered}
D_{x} \vec{b}, D_{x} \vec{G} \in C\left(0, T ; C^{m+1+\alpha}\left(\mathbb{R}^{n}\right)\right), \quad m \geqslant 0, \\
D_{x}^{m+1-j} D_{\vec{w}}^{j} \vec{G} \in L^{\infty}\left(\mathbb{R}^{n} \times 0, T\right), \quad 0 \leqslant j \leqslant m+1, \\
D_{x} \vec{w}_{0} \in C^{m+1+\alpha}\left(\mathbb{R}^{n}\right) .
\end{gathered}
$$

Then there exists a unique solution to 3.1, 3.2 such that

$$
\vec{w}_{t}, D_{x} \vec{w} \text { belong to } C\left(0, T ; C^{m+1+\alpha}\left(\mathbb{R}^{n}\right)\right) .
$$

Proof. A similar result with $m+1=0$ and $C\left(0, T ; C^{m+1+\alpha}\left(\mathbb{R}^{n}\right)\right)$ replaced by $C_{x, t}^{\alpha, \beta}\left(\mathbb{R}^{n} \times(0, T)\right)$ was proved in [8, Lemma 2.2]. The proof of the present lemma for $m+1=0$ is similar and will be omitted. The proof for $m+1>0$ follows by successive differentiation of (3.1) with respect to $x$.

As in Section 1 we shall use the notation

$$
\Omega=\Omega_{0}, \quad \Gamma=\Gamma_{0} .
$$

REMARK 3.1 Later on we shall consider 3.1) with $\vec{b}=\vec{v}$, where $(\vec{v}, p)$ is the solution asserted in Theorems 2.1 and 2.2. Since $\vec{v} \cdot \vec{n}=V_{n}$ on $\Gamma_{t}$, the characteristic curves initiating in $\Omega_{0}$ (or at $\Gamma_{0}$ ) will lie in $\Omega_{t}$ (or on $\Gamma_{t}$ ) for all $t$. Hence the proof of Lemma 3.1 remains unchanged if we replace $\mathbb{R}^{n} \times(0, T)$ by $\bigcup_{0<t<T} \Omega_{t} \times\{t\}$. It will be convenient to consider the solution $\vec{w}$ as a function of $(\xi, t)$ where $\xi \in \Omega$ and $x$ is related to $\xi$ by 2.10 . If we write

$$
\vec{W}(\xi, t)=\vec{w}(x, t),
$$

equation 3.1 takes the form

$$
\vec{W}_{t}+\vec{b} \cdot A \nabla_{\xi} \vec{W}=\vec{G}
$$

where $A=\left(A_{i j}\right)$ and the $A_{i j}$ are defined by 2.14 . Since $\vec{u}_{t}$ and $D_{\xi} \vec{u}$ belong to $C\left(0, T ; C^{m+1+\alpha}(\Omega)\right)$, Lemma 3.1 implies that

$$
\vec{W}_{t}, D_{\xi} \vec{W} \text { belong to } C\left(0, T ; C^{m+1+\alpha}(\Omega)\right) \text {. }
$$


We introduce the space

$$
X^{m+1+\alpha}=\left\{\varphi(\xi, t): \varphi_{t}, D_{\xi} \varphi \text { belong to } C\left(0, T ; C^{m+1+\alpha}(\Omega)\right)\right\}
$$

with norm $\|\varphi\|_{m+1+\alpha}$ defined by 2.19 . For any $M>0$ we set

$$
X_{M}^{m+1+\alpha}=\left\{\varphi \in X^{m+1+\alpha}:\|\varphi\|_{m+1+\alpha} \leqslant M\right\} .
$$

In what follows we shall assume that

$$
\text { the functions } K_{B}(c), K_{Q}(c), K_{R}(c) \text {, and } K_{N}(c) \text { belong to } C^{m+1+\alpha}\left(\mathbb{R}^{1}\right) \text {. }
$$

This implies that

$$
\text { the functions } h(c, r, q), f(c, r, q) \text {, and } g(c, r, q) \text { belong to } C^{m+1+\alpha}\left(\mathbb{R}^{3}\right) \text {. }
$$

We shall also assume that

$$
\Gamma_{0} \in C^{m+3+\alpha}, \quad r_{0} \in C^{m+2+\alpha}(\Omega), \quad q_{0} \in C^{m+2+\alpha}(\Omega)
$$

and that, for some $T_{0}>0$,

$$
\vec{A}(t), \vec{B}(t) \text { are continuously differentiable for } 0 \leqslant t \leqslant T_{0} .
$$

We can now state the main result of the paper.

THEOREM 3.1 If (3.4) (or 3.5) and (3.6), 3.7) hold for some $m \geqslant 0$ and $0<\alpha<1$, then there exists a unique solution of 11.3$)-1.17$ for some time interval $0 \leqslant t \leqslant T$ such that $\Gamma_{t}$ belongs to $C\left(0, T ; C^{m+3+\alpha}\right) \cap C^{1}\left(0, T ; C^{m+2+\alpha}\right)$ and, in Lagrangian coordinates $(\xi, t)$, the function $\vec{u}(\xi, t) \equiv$ $\vec{v}(x, t)$ belongs to

$$
C\left(0, T ; C^{m+2+\alpha}(\Omega)\right) \cap C^{1}\left(0, T ; C^{m+1+\alpha}(\Omega)\right),
$$

and the pressure $p$ and the cell densities $r, q$ belong, in the variables $(\xi, t)$, to

$$
C\left(0, T ; C^{m+1+\alpha}(\Omega)\right) \cap C^{1}\left(0, T ; C^{m+\alpha}(\Omega)\right) .
$$

Proof. The proof is by a fixed point argument. Let $(c, r, q) \in X_{M}^{m+1+\alpha}$. We shall define a mapping

$$
(\hat{c}, \hat{r}, \hat{q})=S(c, r, q)
$$

from $X_{M}^{m+1+\alpha}$ into $X_{M}^{m+1+\alpha}$, for some $m>0$, and prove that it is a contraction, and that the corresponding fixed point yields the solution asserted in the theorem.

Set

$$
\tilde{h}(\xi, t)=h(c(\xi, t), r(\xi, t), q(\xi, t))
$$

By Theorems 2.1 and 2.2 there exists a unique solution $(\vec{u}, \tilde{p})$ of 2.11, 2.2 -2.5 for the function $g(x, t)$ defined as $\tilde{h}(\xi, t)$. We next want to solve the equations

$$
\Delta \hat{c}-\lambda(r+q) \hat{c}=0 \quad \text { in } \Omega_{t}, \quad \hat{c}=\bar{c} \quad \text { on } \Gamma_{t},
$$


and

$$
\begin{aligned}
& \frac{\partial \hat{r}}{\partial t}+\vec{v} \cdot \nabla_{x} \hat{r}=f(\hat{c}, \hat{r}, \hat{q}) \quad \text { in } \Omega_{t}, 0<t<T \\
& \frac{\partial \hat{q}}{\partial t}+\vec{v} \cdot \nabla_{x} \hat{q}=g(\hat{c}, \hat{r}, \hat{q})
\end{aligned}
$$

with

$$
\left.\hat{r}\right|_{t=0}=r_{0},\left.\quad \hat{q}\right|_{t=0}=q_{0} \quad \text { in } \Omega_{0} .
$$

However, it will be more convenient to do this in the Lagrangian variables $(\xi, t)$. Then 3.9 takes the form

$$
L \hat{c}-\lambda(r+q) \hat{c}=0 \quad \text { in } \Omega, \quad \hat{c}=\bar{c} \quad \text { on } \Gamma,
$$

where

$$
L=\sum a_{i j}(\xi, t) \frac{\partial^{2}}{\partial \xi_{i} \partial \xi_{j}}+\sum b_{i}(\xi, t) \frac{\partial}{\partial \xi_{i}}
$$

the $a_{i j}$ are quadratic polynomials in the variables $A_{k \ell}$ introduced in 2.14) and the $b_{i}$ are linear functions in the derivatives $\partial A_{k \ell} / \partial \xi_{n}$. The system (3.10) takes the form

$$
\begin{aligned}
& \frac{\partial \hat{r}}{\partial t}+\vec{u} \cdot A \nabla_{\xi} \hat{r}=f(\hat{c}, \hat{r}, \hat{q}) \quad \text { in } \Omega, 0<t<T, \\
& \frac{\partial \hat{q}}{\partial t}+\vec{u} \cdot A \nabla_{\xi} \hat{q}=g(\hat{c}, \hat{r}, \hat{q}) \quad \text { in } \Omega, 0<t<T,
\end{aligned}
$$

where $A$ is the matrix $\left(A_{i j}\right)$.

Note that the coefficients of $L$ and their $t$-derivatives belong to $C\left(0, T ; C^{m+\alpha}(\Omega)\right)$. Hence, by elliptic estimates,

$$
\hat{c} \in C^{1}\left(0, T ; C^{m+2+\alpha}(\Omega)\right) .
$$

By Lemma 3.1 and Remark 3.1 we then conclude that the system 3.13, 3.11) has a unique solution with

$$
(\hat{r}, \hat{q}) \in C\left(0, T ; C^{m+2+\alpha}(\Omega)\right) \cap C^{1}\left(0, T ; C^{m+1+\alpha}(\Omega)\right) .
$$

Thus $(\hat{c}, \hat{r}, \hat{q}) \in X_{N}^{m+2+\alpha}$ for some $N$, and, as is easily seen, $N$ depends only on $M$.

We now set

$$
S(c, r, q)=(\hat{c}, \hat{r}, \hat{q})
$$

so that the mapping $S$ satisfies

$$
S\left(X_{M}^{m+1+\alpha}\right) \subset X_{N}^{m+2+\alpha}, \quad N=N(M) .
$$

From Remark 1.1 we also easily infer that

$$
\||\hat{c}|+|\hat{r}|+|\hat{q}|\|_{L^{\infty}(\Omega)} \leqslant C_{0}
$$

where $C_{0}$ is a constant independent of $M$.

By standard calculus estimates one can show that for any $\eta>0$ there is a constant $C_{\eta}$ such that

$$
\|w\|_{m+1+\alpha} \leqslant \eta\|w\|_{m+2+\alpha}+C_{\eta}\|w\|_{L^{\infty}}
$$


for any function $w$ in $X^{m+1+\alpha}$. From 3.14, 3.15, we then deduce that

$$
S\left(X_{M}^{m+1+\alpha}\right) \subset X_{M}^{m+1+\alpha}
$$

provided $M$ is sufficiently large. We next prove that $S$ is a contraction mapping in $X_{M}^{m+1+\alpha}$ if $T$ is sufficiently small.

Take any $\left(c_{i}, r_{i}, q_{i}\right)(i=1,2)$ in $X_{M}^{m+1+\alpha}$ and set

$$
c=c_{1}-c_{2}, \quad r=r_{1}-r_{2}, \quad q=q_{1}-q_{2}, \quad \delta=\|(c, r, q)\|_{m+1+\alpha} .
$$

Denote by $\vec{u}_{i}$ the velocity corresponding to $\left(c_{i}, r_{i}, q_{i}\right)$ (according to Theorems 2.1 and 2.2). Then by the estimates of [22] we have

$$
\left\|\vec{u}_{1}-\vec{u}_{2}\right\|_{C\left(0, T ; C^{m+2+\alpha}(\Omega)\right)}+\left\|\vec{u}_{1}-\vec{u}_{2}\right\|_{C^{1}\left(0, T ; C^{m+1+\alpha}(\Omega)\right)} \leqslant C_{1} \delta .
$$

By the arguments used in the proof of Lemma 3.1 (cf. [6]) we can then derive the estimate

$$
\left\|S(c, r, q)-S\left(c_{2}, r_{2}, q_{2}\right)\right\|_{m+2+\alpha} \leqslant C_{1} \delta
$$

with another constant $C_{1}$, so that, by 3.16 ,

$$
\left\|S(c, r, q)-S\left(c_{2}, r_{2}, q_{2}\right)\right\|_{m+1+\alpha} \leqslant \eta \delta+C_{\eta}\||c|+|r|+|q|\|_{L^{\infty}}
$$

for any small $\eta>0$.

If we take the difference of the hyperbolic equations for $r_{1}$ and $r_{2}$, we readily obtain the inequality $|r| \leqslant C T \delta$ for some constant $C$. The same estimate holds for $|q|$, and then from the equation for $c=c_{1}-c_{2}$ we deduce that also $|c| \leqslant C T \delta$ with another constant $C$. Hence the right hand side of 3.18 is bounded by $\eta \delta+C_{\eta} C T \delta$. Taking $\eta=1 / 4$ and $T$ small enough, we conclude that $S$ is a contraction, so that it has a unique fixed point.

The fixed point is not yet a solution of $1.3-(1.17)$ since we still have to eliminate the term $\ell(\vec{v})$ and verify the constraints (1.12), (1.13). But this follows from Theorem 2.3 and Remark 1.2

\section{Further results and open problems}

REMARK 4.1 Theorem 3.1 does not require the special structure (1.7) of the functions $h(c, r, q), f(c, r, q)$, and $g(c, r, q)$. The theorem is valid for general functions $h, f, g$ of $(c, r, q)$ provided we can establish the relation $h(\bar{c}, r, q)=$ const on $\Gamma_{t}$ for all $t$, for the solution of [1.5], (1.6) with constant initial data on $\Gamma_{0}$.

REMARK 4.2 Theorem 3.1 extends to the case where the elliptic equation $(1.8)$ is replaced by the parabolic equation

$$
-\beta c_{t}+\Delta c-\lambda(r+q) c=0 \quad \text { in } \Omega_{t}, t>0(\beta>0),
$$

and $\left.c\right|_{t=0}=c_{0}(x)$ is given. In this case we require that $c_{0}$ belongs to $C^{m+2+\alpha}(\Omega)$ and that the appropriate consistency conditions hold at $\Gamma_{0}$ so that we can use the Schauder estimates.

Theorem 3.1 raises the following questions:

PRoblem 1 For what initial data does there exist a solution of $(1.3)-(1.16)$ for all $t>0$ ?

Problem 2 What will be the asymptotic behavior of such a solution as $t \rightarrow \infty$ ? 
These questions lead us to explore whether stationary solutions to $(1.3)-(1.16)$ do in fact exist. Let us consider the simple case where we have only proliferating cells, that is, $r \equiv 1, q \equiv 0$, and that the proliferating rate is $\mu(c-\tilde{c})$. We shall consider the case where $c$ satisfies a parabolic equation. Then

$$
\begin{aligned}
\nabla p-v \Delta \vec{v}-\frac{1}{3} \nu \nabla \operatorname{div} \vec{v}=0 & \text { in } \Omega_{t}, t>0, \\
\operatorname{div} \vec{v}=\mu(c-\tilde{c}) & \text { in } \Omega_{t}, t>0, \\
-\beta c_{t}+\Delta c-c=0 & \text { in } \Omega_{t}, t>0(\beta>0),
\end{aligned}
$$

the boundary conditions 1.9$]-1.10$ hold,

$$
c=\bar{c} \quad \text { on } \Gamma_{t}, t>0 ;\left.\quad c\right|_{t=0}=c_{0}(x) \quad \text { on } \Omega_{0},
$$

and we take the constraints 1.12 - 1.13 to be

$$
\int_{\Omega_{t}} \vec{v} \mathrm{~d} x=0, \quad \int_{\Omega_{t}} \vec{v} \times \vec{x} \mathrm{~d} x=0 .
$$

We shall denote this problem by $\left(A_{E}\right)$, and its stationary version by $\left(A_{0}\right)$. We make the assumption that $\tilde{c}<\bar{c}$; this ensures that the tumor will not disappear in finite time.

THEOREM 4.1 There exists a unique spherically symmetric stationary solution to problem $\left(A_{0}\right)$, with free boundary $r=R$, and it is given by

$$
\begin{gathered}
\vec{v}=\mu G(r) \vec{x}, \quad p=\bar{p}+\frac{4}{3} \nu \mu c, \\
G(r)=g(r)-g(R), \quad g(r)=\int_{0}^{r} \frac{\mathrm{d} r}{r^{4}} \int_{0}^{r} c^{\prime}(\rho) \rho^{3} \mathrm{~d} \rho
\end{gathered}
$$

where

$$
c(r)=\bar{c} \frac{R}{\sinh R} \frac{\sinh r}{r}
$$

and $R$ is the solution of the equation

$$
R \tanh R=\frac{R}{1+\Lambda R^{2}}, \quad \Lambda=\frac{1}{3} \frac{\tilde{c}}{\bar{c}} .
$$

The constant $\bar{p}$ is determined by the condition

$$
\left.p\right|_{r=R}=\frac{\gamma}{R}+\frac{4}{3} \nu \mu(\bar{c}-\tilde{c}) .
$$

The proof of Theorem 4.1 is by direct calculation. The fact that (4.6) has a unique solution was proved in [17].

PROBLEM 3 Is the stationary solution established in Theorem 4.1 asymptotically stable?

By asymptotic stability we mean that for any initial data near the stationary solution there exists a solution to problem $\left(A_{E}\right)$ for all $t>0$, and the free boundary converges to the sphere $\{r=R\}$ as $t \rightarrow \infty$.

The answer to Problem 3 should depend on the value of $\mu$. For $\mu=0$, the case of viscous droplet, asymptotic stability was proved by Solonnikov [23], Günther and Prokert [20], and Friedman and Reitich [19]. 
In the case of Darcy's law (instead of the Stokes equation), Problem 3 was completely solved by Friedman and $\mathrm{Hu}$ [14], [15]; see also [1]. In this case, there exists a number $\mu_{*}$ such that asymptotic stability holds if $\mu<\mu_{*}$, whereas the stationary solution is linearly unstable if $\mu>\mu_{*}$. Is there a similar result in the case of the Stokes equation?

For the model with Darcy's law it was proved in [9], [18] that there exists an increasing sequence $\left\{\mu_{n}: n \geqslant 2\right\}$ of bifurcation points of symmetry-breaking branches of solutions with free boundary

$$
r=R+\varepsilon Y_{n, 0}(\theta)+\sum_{k=2}^{\infty} \varepsilon^{k} \Lambda_{k}(\theta) \quad(|\varepsilon| \text { small })
$$

where $Y_{n, 0}(\theta)$ is the spherical harmonic of order $(n, 0)$. Clearly $\mu_{2} \geqslant \mu_{*}$, but the case $\mu_{2}>\mu_{*}$ cannot be ruled out. Indeed, such an inequality does occur if $R$ is small, and in that case the value $\mu=\mu_{*}$ corresponds to a Hopf bifurcation [16].

PROBLEM 4 Is there a similar sequence of bifurcation points for the stationary version of the problem 4.1-4.5, 1.9 -1.10?

Problem 5 Compare the values $\mu_{*}, \mu_{2}, \mu_{3}, \ldots$ for the two models, the one with Darcy's law and the other with the Stokes equation.

We note that instead of taking $\mu$ as the bifurcation parameter, we could have taken $\gamma$ as a bifurcation parameter. The two parameters, after scaling, appear as a single parameter $\mu / \gamma$.

Problems 3, 4 deal with the case where all the cells in the tumor are proliferating.

Problem 6 Explore Problems 3, 4 for the general model (1.3)-(1.17).

In the case of Darcy's law, partial results have been proved in [5], [7], [8].

\section{Added in proof}

A solution to Problems 3-5 was recently given in the following articles by A. Friedman and B. Hu:

1. Bifurcation for a free boundary problem modeling tumor growth by Stokes equation. Submitted for publication.

2. Bifurcation from stability to instability for a free boundary problem modeling tumor growth by Stokes equation. Math. Anal. Appl. (2006), to appear.

\section{Acknowledgements}

This work is partially supported by the National Science Foundation upon Agreement No. 0112050.

\section{REFERENCES}

1. Bazaliy, B., \& Friedman, A. Global existence and stability for an elliptic-parabolic free boundary problem: An application of a model of tumor growth. Indiana Univ. Math. J. 52 (2003), 1265-1304. Zbl pre02247562 MR 2010327

2. BYRnE, H. M. The importance of intercellular adhesion in the development of carcinomas. IMA J. Math. Appl. Med. Biol. 14 (1997), 305-323. Zbl 0891.92017

3. BYRne, H. M. A weakly nonlinear analysis of a model of vascular solid tumor growth. J. Math. Biol. 39 (1999), 59-89. Zbl 0981.92011 MR 1705626 
4. Byrne, H. M., \& Chaplain, M. A. J. Modelling the role of cell-cell adhesion in the growth and development of carcinomas. Math. Comput. Model. 12 (1996), 1-17. Zbl 0883.92014

5. Chen, X., Cui, S., \& Friedman, A. A hyperbolic free boundary problem modeling tumor growth: Asymptotic behavior. Trans. Amer. Math. Soc. 357 (2005), 4771-4804. Zbl 1082.35166 MR 2165387

6. Chen, X., \& Friedman, A. A free boundary problem for an elliptic-hyperbolic system: An application to tumor growth. SIAM J. Math. Anal. 35 (2003), 974-986. Zbl 1054.35144 MR 2049029

7. CUI, S., \& FRIEDMAN, A. A hyperbolic free boundary problem modeling tumor growth. Interfaces Free Bound. 5 (2003), 159-182. Zbl 1040.35143 MR 1980470

8. CUI, S., \& FrIEDMAN, A. A free boundary problem for a singular system of differential equations: An application to a model of tumor growth. Trans. Amer. Math. Soc. 355 (2003), 3537-3590. Zbl 1036.34018 MR 1990162

9. Fontelos, M. A., \& Friedman, A. Symmetry-breaking bifurcations of free boundary problems in three dimensions. Asymptotic Anal. 35 (2003), 187-206. Zbl 1054.35145 MR 2011787

10. Franks, S. J. H., Byrne, H. M., King, J. P., Underwood, J. C. E., \& Lewis, C. E. Modelling the early growth of ductal carcinoma in situ of the breast. J. Math. Biol. 47 (2003), 424-452. Zbl 1050.92030 MR 2029006

11. Franks, S. J. H., Byrne, H. M., King, J. P., Underwood, J. C. E., \& Lewis, C. E. Modelling the growth of comedo ductal carcinoma in situ. Math. Med. Biol. 20 (2003), 277-308. Zbl 1039.92021

12. Franks, S. J. H., Byrne, H. M., Underwood, J. C. E., \& Lewis, C. E. Biological inferences from a mathematical model of comedo ductal carcinoma in situ of the breast. J. Theoret. Biol. 232 (2005), 523-543. MR 2125830

13. Franks, S. J. H., \& KInG, J. P. Interactions between a uniformly proliferating tumour and its surroundings: Uniform material properties. Math. Med. Biol. 20 (2003), 47-89. Zbl 1044.92032

14. Friedman, A., \& HU, B. Bifurcation from stability to instability for a free boundary problem arising in tumor model. Arch. Ration. Mech. Anal. 180 (2006), 293-330. MR 2210911

15. Friedman, A., \& HU, B. Asymptotic stability for a free boundary problem arising in a tumor model. J. Differential Equations 227 (2006), 598-639.

16. Friedman, A., \& HU, B. Stability and instability of Liapounov-Schmidt and Hopf bifurcation for a free boundary problem arising in a tumor model. Manuscript submitted for publication.

17. Friedman, A., \& Reitich, F. Analysis of a mathematical model for growth of tumors. J. Math. Biol. 38 (1999), 262-284. Zbl 0944.92018 MR 1684873

18. Friedman, A., \& REITICH, F. Symmetry-breaking bifurcation of analytic solutions to free boundary problems: An application to a model of tumor growth. Trans. Amer. Math. Soc. 353 (2001), 1587-1634. Zbl 0983.35019 MR 1806728

19. Friedman, A., \& Reitich, F. Quasi-static motion of a capillary drop, II: The three-dimensional case. J. Differential Equations 186 (2002), 509-557. Z Zbl pre01891253 MR 1942220

20. GÜNTHER, M., \& PROKert, G. Existence results for the quasistationary motion of a free capillary liquid drop. Z. Anal. Anwendungen 16 (1997), 311-348. Zbl 0888.35140 MR 1459961

21. Pettet, G., Please, C. P., Tindall, M., \& McElwain, D. The migration of cells in multicell tumor spheroids. Bull. Math. Biol. 63 (2001), 231-257.

22. Solonnikov, V. A. On quasistationary approximation in the problem of motion of a capillary drop. Topics in Nonlinear Analysis, J. Escher \& G. Simonett (eds.), Progr. Nonlinear Differential Equations Appl. 35, Basel, Birkhäuser (1999), 643-671. Zbl 0919.35103 MR 1725589

23. Solonnikov, V. A. On the quasistationary approximation in the problem of evolution of an isolated liquid mass. Free Boundary Problems: Theory and Applications I, N. Kenmochi (ed.), Gakkōtosho, Tokyo (2000), 327-342. Zbl 0961.35115 MR 1793044

24. Vainberg, M. M., \& Trenogin, V. A. Theory of Branching Solutions of Non-Linear Equations. Noordhoff, Leyden (1974). Zbl 0274.47033 MR 0344960 